\title{
Hitch Hiker 2.0: a binding model with flexible data aggregation for the Internet-of-Things
}

\author{
Gowri Sankar Ramachandran ${ }^{1 *}$, José Proença ${ }^{1,3}$, Wilfried Daniels ${ }^{1}$, Mario Pickavet ${ }^{2}$, \\ Dimitri Staessens ${ }^{2}$, Christophe Huygens ${ }^{1}$, Wouter Joosen ${ }^{1}$ and Danny Hughes ${ }^{1}$
}

\begin{abstract}
Wireless communication plays a critical role in determining the lifetime of Internet-of-Things (loT) systems. Data aggregation approaches have been widely used to enhance the performance of loT applications. Such approaches reduce the number of packets that are transmitted by combining multiple packets into one transmission unit, thereby minimising energy consumption, collisions and congestion. However, current data aggregation schemes restrict developers to a specific network structure or cannot handle multi-hop data aggregation. In this paper, we propose Hitch Hiker 2.0, a component binding model that provides support for multi-hop data aggregation. Hitch Hiker uses component meta-data to discover remote component bindings and to construct a multi-hop overlay network within the free payload space of existing traffic flows. Hitch Hiker 2.0 provides end-to-end routing of low-priority traffic while using only a small fraction of the energy of standard communication. This paper extends upon our previous work by incorporating new mechanisms for decentralised route discovery and providing additional application case studies and evaluation. We have developed a prototype implementation of Hitch Hiker for the LooCl component model. Our evaluation shows that Hitch Hiker consumes minimal resources and that using Hitch Hiker to deliver low-priority traffic reduces energy consumption by up to $32 \%$.
\end{abstract}

Keywords: Data aggregation, Binding model, Component-based software engineering, Low energy, Component meta data, Middleware, loT

\section{Introduction}

Internet-of-Things (IoT) devices must operate for long periods on limited power supplies and research has shown that wireless communication is the primary source of energy consumption in IoT devices [1]. The lifetime of IoT applications can therefore be increased by minimising radio communication. Data aggregation has been widely applied to tackle this problem [2-4]. Data aggregation is a technique in which multiple messages are combined in to a single datagram, thus reducing radio transmissions and hence the energy consumption of IoT devices. Furthermore in CSMA networks, less frequent transmissions result in fewer collisions and

*Correspondence: gowrisankar.ramachandran@cs.kuleuven.be

1 iMinds-DistriNet, KU Leuven, 3001 Leuven, Belgium

Full list of author information is available at the end of the article therefore retransmissions. This significantly improves the performance of IoT devices.

This paper focuses on lossless data aggregation, through the efficient merging of application traffic flows, rather than algebraic in-network aggregation. Contemporary approaches to lossless data aggregation may be classified as either application dependent or application independent [5]. Application dependent approaches [6-8] support the creation of optimal network-wide data aggregation structures, but restrict the topology of the distributed application. In contrast, application independent approaches $[6,9]$ embed generic aggregation functionality in the underlying network stack, but do not consider the application, and therefore do not achieve optimal performance.

A new approach is needed that allows developers to build custom application communication structures, while providing support for efficient data aggregation. To 
tackle this problem, this paper introduces Hitch Hiker, a lightweight remote binding model with support for multi-hop data aggregation. Hitch Hiker uses the same semantics to configure aggregate data flows as standard bindings, reducing development overhead.

A component binding model specifies how remote software components communicate. Well known examples include Remote Procedure Call (RPC) $[10,11]$ and eventbased communication $[12,13]$. Hitch Hiker extends binding models to distinguish between high- and low-priority bindings. Low-priority bindings use a multi-hop data aggregation overlay network, built from the free payload space of high-priority bindings, and therefore avoid additional radio transmissions between remote components. Using component meta-data, Hitch Hiker constructs a multi-hop data aggregation overlay. The Hitch Hiker binding model allows developers to specify high-priority remote bindings that generate radio transmissions, or lowpriority remote bindings which communicate exclusively using the data aggregation overlay and therefore result in no additional transmissions. By routing low-priority traffic over this data aggregation overlay, Hitch Hiker significantly reduces energy consumption. Furthermore, low-priority bindings provide developers with an elegant way of configuring data aggregation. To the best of our knowledge, Hitch Hiker is the first binding model that provides built-in support for data aggregation.

Our previous short paper on this topic [14] introduced a centralised version of Hitch Hiker, in which multi-hop data aggregation is managed by a single network entity. This paper extends Hitch Hiker [14] by allowing the user to choose between centralised Hitch Hiker or Ad-hoc Hitch Hiker. The Ad-hoc variant of Hitch Hiker eliminates the dependency on the network manager, thereby allowing Hitch Hiker to operate in a fully distributed manner. This allows for the use of multiple meta-managers as supported by LooCI. Ad-hoc Hitch Hiker uses an approach inspired by the well-known Adhoc On-Demand Distance Vector (AODV) [15] routing approach on top of the aggregation overlay for discovering data aggregation routes.

A prototype of Hitch Hiker has been implemented for the LooCI component model [13] running on the Contiki OS [16] and for the OMNeT++ [17] simulator. Our evaluation using two real-world case studies show that: (i.) the resource consumption of Hitch Hiker is minimal and (ii.) by using Hitch Hiker to transmit low-priority traffic, energy consumption is significantly reduced.

The remainder of this paper is structured as follows. Section 2 reviews related work. Section 3 introduces the Hitch Hiker-2.0 binding model. Section 4 explains the route discovery process of infrastructure Hitch Hiker. The ad-hoc mode of Hitch Hiker is explained in Section 5. Section 6 discusses the route maintenance schemes of Hitch Hiker. Section 7 describes our case study applications. Section 8 introduces and evaluates prototype implementations of Hitch Hiker-2.0. Finally Section 9 concludes and discusses directions for future work.

\section{Related work}

We draw upon two streams of prior work. Section 2.1 discusses related work in the area of data aggregation. Section 2.2 discusses contemporary component and binding models. We then discuss opportunities for applying data aggregation in component bindings in Section 2.3.

\subsection{Data aggregation schemes}

He et al. [5] describe two classes of data aggregation approach: Application Dependent Data Aggregation (ADDA), which requires knowledge of application-level traffic flows and Application Independent Data Aggregation (AIDA) which performs aggregation in a generic fashion without application-specific information. We discuss both classes of aggregation in Sections 2.1.1 and 2.1.2, respectively.

\subsubsection{Application dependent data aggregation}

ADDA approaches use network-wide application information to optimise the manner in which information is collected and routed across the network. These efforts focus upon the network and application layers of the communication stack.

At the Network Layer, Intanagonwiwat et al. introduce Directed Diffusion [6], which provides data-centric routing, in-network caching and aggregation. To realise these features, Directed Diffusion provides a common data representation. Entities that request data register an interest in a particular data type at a certain network location, which causes a conceptual gradient to be established between sources and requesters, data is then drawn down these gradients from sources to requesters. As data travels down these routes, it is aggregated and cached.

At the Application Layer, Madden et al. contribute the Tiny AGgregation (TAG) [7] service, which allows users to specify SQL-like queries, which are multicast to relevant sensor nodes using a tree that is rooted at the base-station. As responses travel towards the root, developer-specified aggregation functions may be applied to data at each hop. Heinzelman et al. contribute the Low-Energy Adaptive Clustering Hierarchy (LEACH) protocol [8], which creates a clustered network structure that provides inherent support for aggregation and energy balancing during data collection. SPEED [18] and SPIN [19] extend application layer aggregation approaches to consider current energy levels when configuring aggregation and routing functionality. Asemani et al. [20] contribute LAG, which aims to create a data aggregation route towards the sink by taking into account the energy levels of nodes and their hop 
depth in the network. LAG uses learning automata to update the route as the energy levels of the nodes change during run-time.

Network-flow based data aggregation protocols [21, 22] take an orthogonal approach, modelling the sensor network as a graph and, based upon application-level traffic flows, calculating and configuring an optimal aggregation structure. Kapakis et al. [21] contribute MDLA, a network-flow based approach to achieving maximum network lifetime using linear programming and constraints. Xue et al. [22] contribute MaxLife, a commodities-inspired algorithm, wherein a commodity models the data that is generated by a sensor node and delivered to a base station. MaxLife is capable of calculating optimal data aggregation structures and thereby extending network lifetime. Voulkidis et al. [23] contribute a game-theoretic approach to reduce the number of transmissions. This approach estimate the spatial correlation of the sensor data and optimises the transmissions based on the spatial relationship. Xiang et al. [24] contribute a data aggregation approach based on compressed sensing, which uses diffusion wavelets to account for the spatial and temporal correlations. Network-flow based approaches offer efficient calculation of an optimal data aggregation structure, for static networks where networkwide data flows are known, but these approaches are unsuitable for dynamic networks which support runtime reconfiguration.

From the above application dependent data aggregation approaches, it can be seen that contemporary approaches are either inherently static as in network flow models [6], or otherwise restrict developers to a single application interaction model [7] or routing topology [21, 22]. In contrast, application-independent approaches provide a more generic aggregation approach, discussed below in Section 2.1.2.

\subsubsection{Application independent data aggregation}

AIDA schemes provide a one size fits all approach to data aggregation that is independent of application requirements. These approaches typically operate at the network and data link layer.

At the Network Layer, well known approaches to aggregation include the Shortest Path Tree (SPT), wherein a single, network-wide aggregation tree is centrally calculated and configured and the Greedy Incremental Tree (GIT) which approximates a shortest path tree, but is constructed in an incremental and decentralised fashion [25]. However, these approaches are poorly suited to Wireless Sensor Networks (WSN) scenarios where energy resources are unevenly distributed. Aonishi et al. contribute Adaptive GIT [9] to address this problem. Intanagonwiwat et al. contribute the Centre at the Nearest Source (CNS) [6] scheme, wherein the responsibility for data aggregation is assigned to the node which is a source of that data type and closest to the destination.

Leandro et al. [26] contribute DRINA, a lightweight and reliable routing approach for in-network aggregation. DRINA follows a cluster-based approach and builds a shortest path tree to sink. Nodes in each cluster forward the sensed data to the cluster head, which then relays the data to the sink. This solution relies on a dedicated node to perform data aggregation, which means all nodes in the cluster must transmit the sensed data to the cluster head. Jongsoo et al. [27] propose Lump to perform Quality-of-Service aware aggregation for heterogeneous traffic. Lump prioritises packet based on the latency requirements. Lump maintains a queue for each next-hop address, where it stores the packets. Lump uses a periodic send-timer, which raises the priority level of the packets on each timer event. Whenever the priority is raised to the highest level, all packets in the queue are aggregated and transmitted.

At the Data Link Layer, He et al. contribute AIDA [5], which takes advantage of queuing delay and the broadcast nature of wireless media to implement application independent data aggregation. AIDA aggregates multiple packets into single frames prior to transmission, resulting in significant savings in terms of energy and latency. While AIDA uses data from the network layer, it treats the application layer as a black box and therefore cannot exploit patterns in application traffic flows. Furthermore, as AIDA operates at the data link layer, it is unable to perform multi-hop data aggregation.

\subsection{Remote component binding models}

Hitch Hiker combines aggregation with a lightweight remote binding model. In this section, we review component binding models and discuss opportunities for aggregation. Contemporary remote binding models typically offer either event-based or Remote Procedure Call (RPC) semantics.

RPC-based binding models allow remote functionality to be called using the same semantics as local procedures, thus lowering the overhead on component developers. RPC-based models are request-reply and therefore bidirectional in nature. In the case of resource-rich sensing systems, Remote Method Invocation (RMI) [10] has been used to provide reliable RPC-based bindings for Java component models such as OSGi [28] and RUNES [29]. In a WSN context, May et al. [11] extend NesC [30] with support for unicast and anycast RPC calls, wherein exactly one neighbouring node responds to the call. Where component models support remote reconfiguration, bindings may be modified at runtime.

Event-based binding models provide simple unidirectional communication between software modules. Eventbased approaches are attractive in resource-constrained 
scenarios, as they are lightweight and do not cause software modules to block while waiting for responses as in RPC. The Active Messages [12] protocol provides remote bindings for the $\mathrm{NesC}$ [30] component model. A unique reference to an application handler is embedded in each active message and is used to dispatch incoming messages to the appropriate handler component. As NesC does not allow for runtime reconfiguration, bindings are fixed at development time. The LooCI binding model [13] provides unreliable event-based binding using a decentralised publish-subscribe event bus communication medium. In contrast to Active Messages, LooCI supports multi-model bindings allowing for the modelling of one-to-one, oneto-many, many-to-one and many-to-many relationships. Additionally, unlike Active Messages, LooCI bindings may be remotely modified at runtime in order to enact reconfiguration.

Considering opportunities for cross-layer optimisation, all of the binding models discussed above $[10,30]$ provide explicit meta-data that can be used to determine traffic flows and therefore optimise aggregation functionality. Despite this opportunity, current component models typically treat the network layer and below as a black box, resulting in suboptimal communication.

\subsection{Opportunities for data aggregation}

There are a number of advantages to embedding data aggregation support in a component binding model:

Flexible Network Topologies: ADDA approaches to data aggregation such as TAG [7] and Directed Diffusion [6] enforce a single network topology, which may be suboptimal for some application scenarios. In contrast, components can be remotely bound together to form distributed component graphs with flexible network topologies.

Support for Multiple Applications: WSN are increasingly required to simultaneously support multiple applications. Contemporary ADDA approaches are poorly suited to multi-application scenarios as they enforce a single routing structure across multiple applications with different networking requirements. In contrast, component bindings can be used to create a specific network topology for each application.

Appropriate Separation of Concerns: ADDA approaches require that the lower layers of the network stack be concerned with application-level data flows [5]. This means that aggregation protocols must be updated whenever new data types are introduced. In contrast, components provide externally visible meta-data that describes data flows via bindings. This allows aggregation functionality to evolve along with the application components during software reconfiguration.

Application-Optimised Aggregation: AIDA approaches such as CNS [6] and AIDA [5] are unaware of application data flows and therefore would be expected to perform sub-optimally in comparison to application dependent approaches. In contrast, component-binding meta-data provides a means to optimise generic aggregation functionality to suit a specific application.

By using component binding meta-data to build a multihop aggregation network, Hitch Hiker combines the key benefits of application dependent aggregation (i.e. an optimised aggregation approach) with those of application independent aggregation (i.e. flexible networking and a more appropriate separation of concerns). The following section describes the Hitch Hiker binding model.

\section{The Hitch Hiker 2.0 binding model}

Hitch Hiker 2.0 extends the previous version of Hitch Hiker, which is reported in [14]. In Hitch Hiker, the bindings are classified as either high or low priority bindings. This classification allows Hitch Hiker to support data aggregation by appending low-priority data in the overlay network created using the unused payload space of high-priority transmissions. Hitch Hiker uses meta data provided by component bindings to create a multi-hop data aggregation overlay. To support end-to-end routing of low-priority traffic, Hitch Hiker performs route discovery on multi-hop overlay network using a central meta-manager.

Hitch Hiker 2.0 expands the previous version of Hitch Hiker [14] with Ad-hoc Hitch Hiker, which does not rely on central meta-manager to discover the data aggregation overlay. Ad-hoc Hitch Hiker uses an approach inspired by AODV for route discovery.

This section describes the design of the Hitch Hiker binding model and its associated network stack. Section 3.1 provide background on LooCI, which is extended with Hitch Hiker. Section 3.2 introduces prioritised bindings. Section 3.3 describes how route information is extracted from bindings. Sections 3.4 and 3.5 describe the Hitch Hiker network stack.

\subsection{The loosely-coupled component infrastructure}

The Loosely-coupled Component Infrastructure (LooCI) [13] is a platform-independent component model and supporting middleware targeting networked embedded systems. The LooCI middleware is open-source and ports are available for embedded operating systems such as Contiki [16], Squawk [31] and Android. LooCI is a representative example of a runtime reconfigurable component model for the IoT, with which we have extensive experience. Hitch Hiker extends the basic LooCI component model to support priority-based multi-hop data aggregation. The remainder of this subsection provides a basic overview of the relevant features of LooCI.

Components LooCI components are individually deployable units of functionality. They are managed by 
creating an instance of the basic LooCI meta-model, described in [13], using a simple component declaration and communication API consisting of required and provided interfaces. LooCI is language-agnostic and components may be implemented in $\mathrm{C}$ or Java, allowing developers to exploit language-specific features while providing standardised encapsulation, discovery and lifecycle management. All messages that travel across component interfaces are hierarchically typed as described in [32]. Components may also declare properties that allow for inspection and customisation of component behaviour through externally accessible name/value tuples.

Communication All LooCI components communicate over a fully distributed 'event bus' spanning the entire network. The event-bus is an asynchronous event-based communication medium that follows a decentralised topic-based publish-subscribe model. Local and remote bindings are established by creating new subscription relationships, supporting one-to-one, many-to-one, and one-to-many bindings (as specified in [13]). Hitch Hiker extends the binding model of LooCI with support for data aggregation. Hitch Hiker classifies LooCI bindings as high-priority and low-priority, and this classification allows Hitch Hiker to support data aggregation by appending low-priority data in the overlay network created using the unused payload space of high-priority transmissions.

Reconfiguration LooCI components are connected to the middleware runtime installed on every device. Each component declares its human-readable name, its required interfaces (i.e. services) and provided interfaces (i.e. dependencies). A reconfiguration engine manages the basic meta-model and supports reflective operations using both local or remote API calls.

Meta Manager LooCI applications are deployed, inspected, and configured by manager nodes. In principle, any LooCI node may serve as a manager and a network may have multiple managers. The manager interacts with the nodes by using the reflection API to inspect and reconfigure LooCl's meta-model. Hitch Hiker uses a single meta manager in infrastructure mode for the creation of data aggregation overlay, which is a major restriction. Hitch Hiker 2.0 supports Ad-hoc Hitch Hiker, which operates with either multiple or no manager at all.

\subsection{Prioritised bindings}

Figure 1 shows a small part of the smart building case study, evaluated later in this paper. Here, a temperature component, deployed on sensor node $N_{1}$, samples temperature data once every $30 \mathrm{~s}$ and sends the data to the comfort level component. The comfort level component on $N_{2}$, analyses sensor data, and sends the result to a manager located on $N_{3}$ every $30 \mathrm{~s}$. These three components communicate via standard bindings, depicted as - -)- For the remainder of this paper, we refer the standard bindings as high-priority bindings. LooCI provides bindTo and bindFrom calls to create high-priority bindings.

Hitch Hiker introduces the concept of low-priority bindings, depicted as $\cdots 0$ ) $\cdots$ in Fig. 1. Low-priority bindings are used by non-critical applications, the definition of which is left to the developer. In principle, the developer should use low-priority bindings for traffic that can tolerate long latencies. Hitch Hiker provides bindHHTO and bindHHFrom calls to create low-priority bindings. In our example, a node monitor component deployed on $N_{1}$ is connected to a node alert component deployed on $N_{3}$ via a low-priority binding. The use of a low-priority binding indicates that the developer is willing to trade communication performance for energy efficiency. Low-priority bindings are realised in Hitch Hiker by routing messages via the data aggregation overlay network, referred to as Hitch Hiker network.

High-Priority Bindings Hitch Hiker extends the eventbased LooCI binding model described in Section 3.1. LooCI bindings are: event-based, unidirectional, and unreliable. Conceptually, a LooCI binding is a connection between a source and a destination component, with an associated data type and a reference to the network link that connects the nodes hosting the two components. Table 1 shows the list of well-known LooCI binding types and their payload sizes.

Definition 1 (Binding). $A$ binding is a tuple $b=$ $\left\langle C_{s}, C_{d}\right.$, Type, Link $\rangle$, where $C_{s}$ is the source component, $C_{d}$ is the destination component, Type is the type of the events sent through the binding, and Link is the remote connection

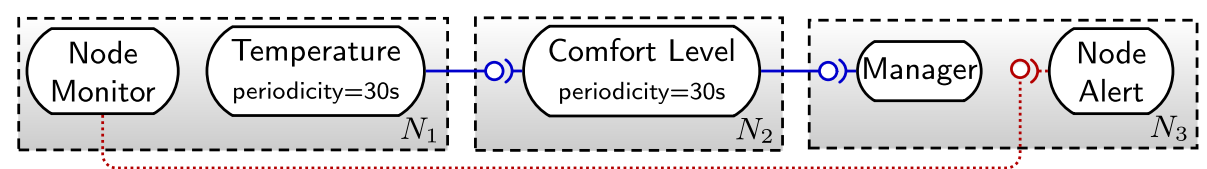

Fig. 1 Application view of a deployed application 
Table 1 List of LooCl binding types and their payload sizes

\begin{tabular}{lll}
\hline Components & Binding type & Payload size (in bytes) \\
\hline Temperature sensor & TEMP & 5 \\
Light sensor & LIGHT & 3 \\
Moisture sensor & MOISTURE & 3 \\
Air quality sensor & AIRQUALITY & 8 \\
PIR sensor & PIR & 4 \\
RFID reader & RFID & 8 \\
Door sensor & DOOR & 3 \\
Buzzer & BUZZER & 3 \\
\hline
\end{tabular}

between the nodes where the binding is deployed, defined below.

Definition 2 (Remote Connection). A remote connection is a tuple $\ell=\left\langle N_{s}, N_{d}, M T U, B w, D\right\rangle$ describing the communication channel between two network nodes, where $N_{s}$ is the source node, $N_{d}$ is the destination node, $M T U$ is the maximum transmission unit between $N_{s}$ and $N_{d}, B w$ is the bandwidth of the remote connection, and $D$ is the expected delay of the remote connection.

A LooCI binding is realised as an outgoing binding entry on the sending node and an incoming binding entry on the receiving node, which is established by issuing bindTo and bindFrom calls to the sender and receiver, respectively. These bindings are stored in a binding table which is used to dispatch events. LooCI bindings are created at runtime after the deployment of the involved components. High-priority binding is mediated by the transmission of a event using the network stack of the host operating system.

The binding from the temperature component is formally represented as 〈Temperature,Comfort Level,Temp, $\ell\rangle$, where the associated remote connection $\ell=\left\langle N_{1}, N_{2}, 127 \mathrm{~B}, 250 \mathrm{kbps}, 0.1 \mathrm{~s}\right\rangle$.

Low-Priority Bindings Hitch Hiker introduces the concept of a low-priority binding, depicted as …… in Fig. 1. In our example, a node monitor component gathers the local node status and transmits this data to a node alert component running on a server. We selected node monitoring as an example of a low-priority application because this functionality is less important than the core WSN mission of gathering environmental data and this application data can tolerate delay. However, it should be noted that developers are free to define which components are high-priority and lowpriority in their application context. In our example (Fig. 1), the low priority binding that connects the node monitor to the node alert component is formally represented as $\left\langle\right.$ Node Monitor, Node Alert, Status, $\left.\ell_{\text {status }}\right\rangle$, where Status is the event type containing node status information and $\ell_{\text {status }}$ is the remote connection of the overlay network.

High-priority and low-priority bindings have an identical set of artefacts: a source component, destination component, data type (Definition 1) and a remote connection (Definition 2). Low-priority bindings are realised in LooCI by adding a separate set of binding tables to each node.

The overlay routes necessary to support low-priority bindings are established reactively, as it required to support low-priority bindings.

\subsection{Component model probe extracts network data}

The component model probe extracts data from the highpriority application to create the remote connections of the Hitch Hiker network. It intercepts binding acknowledgment messages containing the source component $C_{s}$, the source node $N_{s}$, the destination node $N_{d}$, and the binding Type, and builds a remote connection for the Hitch Hiker network. Recall that a remote connection is formally a tuple $\left\langle N_{s}, N_{d}, M T U, B w, D\right\rangle$ (Definition 2). The $M T U$ is calculated based on the event type, which has an associated payload size, as shown in Table 1. Hitch Hiker extracts periodicity information by querying source components for their periodicity property using the standard LooCI API. Hitch Hiker distinguishes between periodic and non-periodic components: the former send values at a fixed rate (e.g., a temperature reading every $10 \mathrm{~s}$ ), and the latter exhibit unpredictable behaviour (e.g., an alert generated when a window is opened).

Formally, we write $\Pi(C)$ to denote the periodicity of a component $C$, defined below, which returns the special symbol $\perp$ when $C$ is non-periodic.

$$
\Pi(C)= \begin{cases}r & \text { if } C \text { is periodic with rate } r \\ \perp & \text { otherwise }\end{cases}
$$

Based upon the information intercepted in the binding acknowledgment-the source component $C_{S}$, the source node $N_{s}$, the destination node $N_{d}$, and the source Typeand the periodicity $\Pi\left(C_{s}\right)$, the probe calculates the remote connection for the Hitch Hiker network as follows.

1. Get the payload size $p s$ associated with the Type.

2. Get the MTU $m$ of the remote connection between the source $\left(N_{s}\right)$ and destination $\left(N_{d}\right)$.

3. Define hd to be the size of the headers used by the data-link, network and transport layers of the host protocol stack.

4. Define $M T U_{H H}$ to be the unused payload size, calculated as $m-p s-h d$. 
5. If $\Pi\left(C_{s}\right)=\perp$ then return the remote connection $\left\langle N_{s}\right.$, $\left.N_{d}, M T U_{H H}, \perp, \perp\right\rangle$, otherwise return the remote connection

$$
\left\langle N_{s}, N_{d}, M T U_{H H}, M T U_{H H} / \Pi\left(C_{s}\right), \Pi\left(C_{s}\right)\right\rangle .
$$

The component model probe reveals the remote connection between a source node $N_{s}$ and a destination node $N_{d}$ with a free payload space of $M T U_{H H}$. The probe also reveals the delay or the time-interval between two successive transmissions as $\Pi\left(C_{s}\right)$. For the example application shown in Fig. 1, the remote connection between node $N_{1}$ and $N_{2}$ has a free payload space of $75 \mathrm{~B}$ (excluding the temperature data and the overhead added by the other layers) with a delay of $30 \mathrm{~s}$. This connection meta data is used for the configuration of Hiker routing protocol and it is discussed in Section 3.5.

\subsection{Hitch medium access control (MAC) protocol}

Figure 2 shows the Hitch Hiker network stack for a single embedded sensor node, with each layer numbered according to the 5-layer Tanenbaum reference model [33]. The Hitch Hiker protocol stack is composed of two protocols the Hitch MAC protocol and the Hiker routing protocol. The former is described below and the latter in the following subsection.

Hitch is a virtual MAC protocol that manages and provides access to the data aggregation overlay links. The Hitch MAC protocol is implemented as an independent module, which allows Hitch to be used with third-party routing protocols at the aggregation overlay level.

Link Data Structures Hitch manages the set of virtual data links that are available on each sensor node. Each virtual data link maps to a remote overlay connection (Definition 2) that may be multi-hop and is composed of: a destination address, MTU, delay and bandwidth. Virtual links are created by the probe and may be accessed through the HitchAPI, available online: http://goo.gl/ $7 \mathrm{~m} 2 \mathrm{nwN}$.

A First In First Out (FIFO) queue is maintained per link where packets are buffered until they can be aggregated with high-priority traffic and dispatched. If the buffer reaches its capacity, the oldest frame in the queue is discarded, resulting in packet loss. Hitch is a best-effort protocol, which provides no reliability guarantees. Where reliability is required, it should be implemented by the upper layers.

Aggregation The Hitch protocol intercepts outgoing packets as they are passed to the host MAC protocol, and this protocol does not violate the security requirements of the host MAC protocol. If the virtual link queue associated with the destination of an intercepted packet is not empty, the available payload size is filled with packets from the queue, until either the available payload space is exhausted or the buffer is empty. The modified

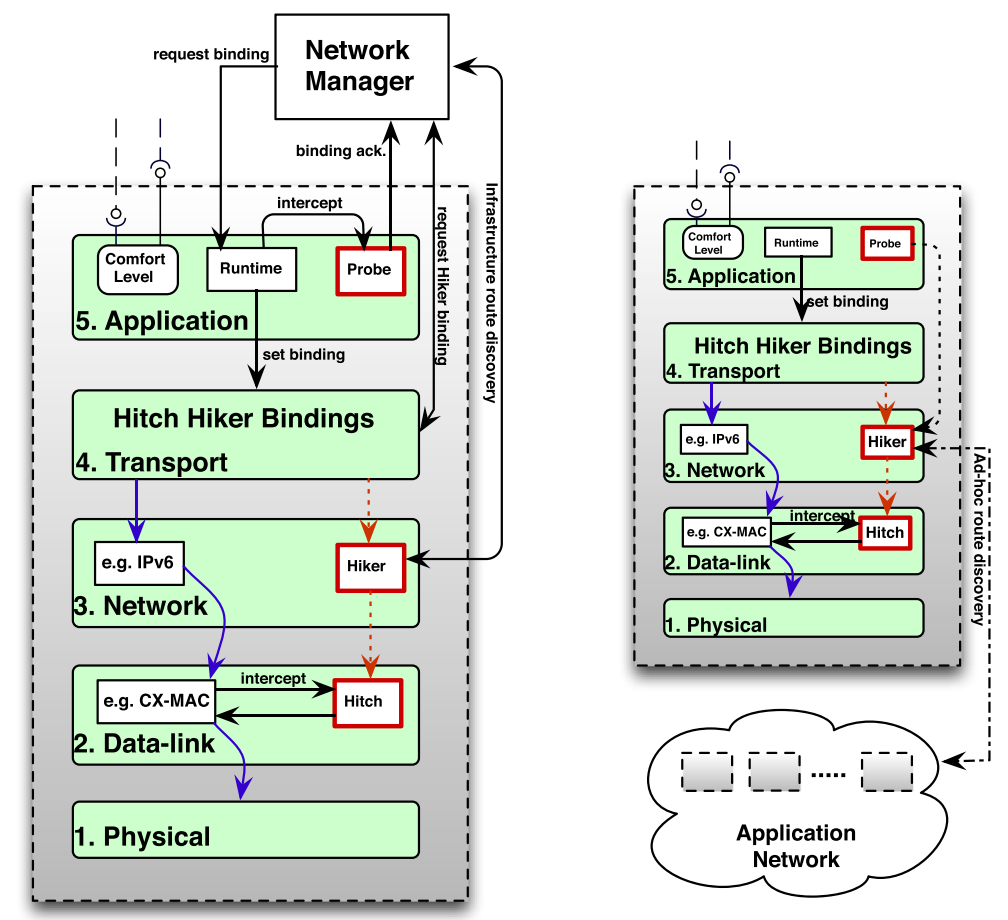

Fig. 2 Architecture of a Hitch Hiker node: Infrastructure Hitch Hiker (left) and Ad-hoc Hitch Hiker (right) 
packet is then returned to the host MAC protocol to be transmitted.

Disaggregation The Hitch protocol intercepts incoming frames in the host MAC protocol, and disaggregates all encapsulated Hitch packets. The disaggregated packets are then passed to the network layer, while the original frame is passed back to the host data link protocol. And, it operates within boundary of the host MAC protocol.

\subsection{Hiker network protocol}

Hiker is a multi-hop routing protocol that operates efficiently with the Hitch data link protocol.

Route Data Structures Hiker maintains a minimalist routing table on each node. This routing table begins empty, and routes are reactively configured by the network manager to support low-priority bindings. Each route is comprised of a remote destination, the virtual link that represents the next hop on the route to this address and a route-MTU which denotes the maximum packet size that can traverse the complete route.

Routing When an incoming Hiker packet is received, the destination field of the packet is checked. If the destination is the local sensor node, it is passed to the transport layer. If the destination matches a known route, it is transmitted on the appropriate link using the transmit (frame, link) method of Hitch. If no route is known, the packet is discarded. Sections 4 and 5 explains the route discovery process of Hitch Hiker in infrastructure and ad-hoc mode, respectively.

Definition 3 (Route). A route is a multi-hop remote connection (Definition 2) obtained by composing a non-empty sequence of remote connections, such that for every consecutive remote connections $\ell$ and $\ell^{\prime}$ the destination node of $\ell$ matches the source node of $\ell^{\prime}$. Given a sequence of $n$ remote connections:

$$
\begin{aligned}
& \left\langle N_{s, 1}, N_{d, 1}, M T U_{1}, B w_{1}, D_{1}\right\rangle, \ldots \\
& \left\langle N_{s, n}, N_{d, n}, M T U_{n}, B w_{n}, D_{n}\right\rangle
\end{aligned}
$$

its composition yields the route $\left\langle N_{s}, N_{d}, M T U, B w, D\right\rangle$, where

$$
\begin{array}{lll}
N_{s}=N_{s, 1} & M T U=\min _{i=1}^{n} M T U_{i} \\
N_{d}=N_{d, n} & B w=\min _{i=1}^{n} B w_{i}
\end{array} \quad D=\sum_{i=1}^{n} D_{i} .
$$

Hiker Packet Encapsulation Figure 3 shows a host packet that is aggregated with multiple Hitch Hiker $(\mathrm{HH})$ packets. Each encapsulated $\mathrm{HH}$ packet has a $2 a+1$ byte header, where $a$ is the length of a network address. One additional byte is used to represent the length of the payload that follows. We use 6LowPAN IPv6 address shortening [34], resulting in $2 \mathrm{~B}$ addresses in our case-studies.

\section{Infrastructure Hitch Hiker}

In infrastructure mode, Hiker assumes that a single LooCI network manager is running for the entire network, as shown in Fig. 2. This network manager enacts all management and reconfiguration. This information is exploited to create the data aggregation overlay network as follows:

1. Overlay links are discovered based upon extended binding acknowledgements. This information is provided by the component model probe as described in Section 3.3.

2. The network manager assembles discovered overlay links to form a network graph, wherein each link is labelled with its associated delay, MTU and bandwidth.

3. When the user requests the establishment of a low-priority binding $b$ :

(a) The graph is pruned to remove all links which have an insufficient MTU to support the specified data type.

(b) The Dijkstra algorithm is used to calculate the shortest path between the source and destination, using either delay or bandwidth as the link cost. Our evaluation uses delay as the link cost.

(c) The network manager configures the shortest path overlay route, or responds with an exception where no overlay route is possible.

(d) Finally, the network manager configures the route required by the low-priority binding $b$, by sending route-creation messages to all involved nodes.

\begin{tabular}{|l|l|l|c|c|c|c|}
\hline $\begin{array}{l}\text { Frame } \\
\text { header frame }\end{array}$ & $\begin{array}{l}\text { Netw. } \\
\text { header }\end{array}$ & Payload & $\begin{array}{c}\mathrm{HH} \\
\text { header }_{1}\end{array}$ & $\begin{array}{c}\mathrm{HH} \\
\text { payload }_{1}\end{array}$ & $\begin{array}{c}\mathrm{HH} \\
\text { header }_{2}\end{array}$ & $\begin{array}{c}\mathrm{HH} \\
\text { payload }_{2}\end{array}$ \\
\hline
\end{tabular}

Fig. 3 Hiker packet; darker background captures the low-priority aggregated data 
Figure 4 shows the networked interactions that are required to create both the high-priority and low-priority bindings for the running example shown in Fig. 1. In the interests of clarity and brevity, binding and route data shows only the network end-points.

Steps (1)-(5) The network manager receives a request to establish high-priority bindings to connect the Temperature, Comfort Level, and Manager components. The network manager then enacts this request by issuing standard LooCI bindTo and bindFrom commands that establish the required binding table entries. The associated binding acknowledgements inform the network manager of newly available overlay routes.

Steps (6)-(8) The network manager receives a request to establish a low-priority binding to connect the Node Monitor and the Node Alert components. To support this binding, Hitch Hiker configures an overlay route between the nodes $N_{1}$ and $N_{3}$ (Definition 3 ).

Steps (9)-(10) The network manager establishes the lowpriority binding by issuing the required bindHHTo and bindHHFrom method calls, which establish the necessary entries in the Hitch Hiker wiring tables.

Since this mode of Hitch Hiker operate with a single network manager, the creation of Hitch Hiker bindings require binding meta data from the entire application network. In principle, any Hitch Hiker node in the network can create or remove bindings. In order to increase the flexibility of Hitch Hiker and to allow Hitch Hiker to operate with either multiple managers or no managers, we extend Hitch Hiker with support for decentralised route discovery, which is explained in Section 5.

\section{Ad-hoc Hitch Hiker}

In ad-hoc mode, Hiker routes are discovered in a fully decentralised manner. This allows Hiker to operate in networks with multiple managers or no managers at all, as shown in Fig. 2. The binding request can come from any node in the application network, and the Hiker of the source node self-discovers a overlay route, unlike
Infrastructure Hitch Hiker. To realise this, Hiker reimagines the well-known Ad-hoc On-Demand Distance Vector (AODV) routing approach [15]. To find overlay routes on top of bindings as opposed to at the network layer. In this mode, Hiker discovers the route as follows:

1. When the user requests the establishment of a low-priority binding $b$, a route-discovery message is flooded across the data aggregation overlay as shown in Fig. 5.

2. The source node broadcasts a discovery message containing the destination address, required MTU, a sequence number and a Time to Live (TTL) on all links provided by the Hitch MAC protocol, as indicated in Fig. 5. And, the source node waits for NET_TRAVERSAL_TIME seconds for routecreation message. If the route-creation message is not received within NET_TRAVERSAL_TIME, then the source node notifies the developer that there is no route between the source and destination, and the low-priority binding is not accepted. Section 6 explains the error handling schemes of Hitch Hiker.

3. All nodes receiving a route-discovery message decrement the TTL, add the sequence number and source link to their cache and re-broadcast the discovery message, discarding the message when the TTL reaches zero, or the available MTU is insufficient, or if the sequence number was previously observed.

4. When the destination node receives the route-discovery message as presented in Fig. 5, it establishes a route $r$ by responding with a route-creation message as follows:

(a) The first route-discovery message received by the destination denotes the shortest path between the source and the destination nodes. The destination node forwards a routecreation message back to the link on which the discovery message was received. This message contains the matching sequence number and the address of the destination node.

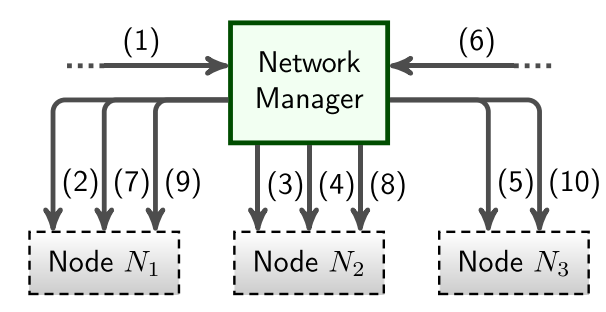

(1) Request HP bindings

(2) bindTo $\quad N_{1} \rightarrow N_{2}$

(3) bindFrom $\quad N_{1} \rightarrow N_{2}$

(4) bindTo $\quad N_{2} \rightarrow N_{3}$

(5) bindFrom $N_{2} \rightarrow N_{3}$

(6) Request LP bindings

(7) addHHRoute $N_{1} \rightarrow N_{2}$

(8) addHHRoute $N_{2} \rightarrow N_{3}$

(9) bindHHTo $\quad N_{1} \rightarrow N_{3}$

(10) bindHHFrom $N_{1} \rightarrow N_{3}$

Fig. 4 Network view - configuration of bindings and routes for Infrastructure Hitch Hiker 
(b) On receipt of a route-creation message, each intermediate node adds a routing table entry mapping the specified destination address to the link on which the route-creation message was received.

(c) Following the creation of a routing table entry, the intermediate node checks its cache and forwards the route creation message on the link via which the original route-discovery was received. Step 2 repeats until the route is fully established.

As can be seen from the process described above, in adhoc mode, Hitch Hiker requires no supporting infrastructure. However, the use of flooding increases the overhead and latency of route discovery in comparison to infrastructure mode. Figure 5 shows the networked interactions that are required to create low-priority bindings for the running example shown in Fig. 1 using AODV routing approach.

\section{Route errors and maintenance}

Whenever a user creates a Hitch Hiker binding as described in Section 3, route discovery executes as described in Sections 4 and 5 . If a low priority route was successfully created, Hitch Hiker returns true together with the performance properties of the route as listed in Definition 3, i.e. route MTU, latency and bandwidth. If the route was not created, Hitch Hiker returns false and the performance properties of the best available route, if one exists. Based upon this information, the developer may choose to (i.) abandon the binding, (ii.) modify the binding to work within available Hitch
Hiker network capacity or (iii.) establish a high-priority binding.

\subsection{Impact of reconfiguration}

Hitch Hiker-2.0 builds on top of LooCI component model, which provides support for run-time reconfiguration of application. Such run-time reconfigurations may result in deployment of new components or the removal of existing components. In addition, the reconfiguration may also change the existing bindings in the application network. These reconfigurations disrupts the existing data aggregation overlay and might invalidate the existing routes of low-priority bindings.

In infrastructure mode, when the manager receives a reconfiguration command that invalidates a route, it removes the old route and then execute the route discovery process to find a replacement route. If no replacement route exists, an exception is generated. In adhoc mode, a mote that receives a reconfiguration command, which impacts a Hitch Hiker route will flood a route-remove message with the sequence number of the matching route. All motes that receive this message will remove the route, causing the source node to re-run the route discovery process.

\section{Case study applications}

We validate the performance of Hitch Hiker-2.0 in two representative application scenarios that are realised using the LooCI component model and prioritised bindings. Section 7.1 describes a low data rate multi-hop static smart office sensor network, while Section 7.2 describes a high data rate one-hop mobile robot sensor network.

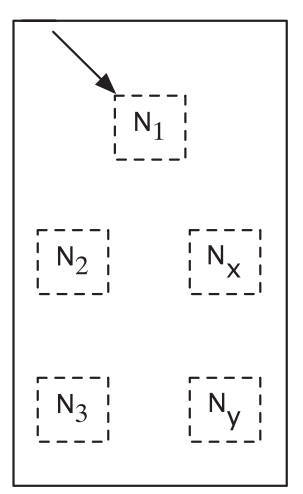

1

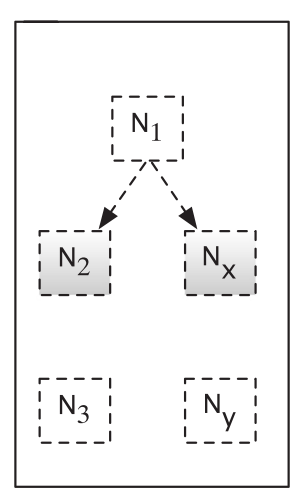

2

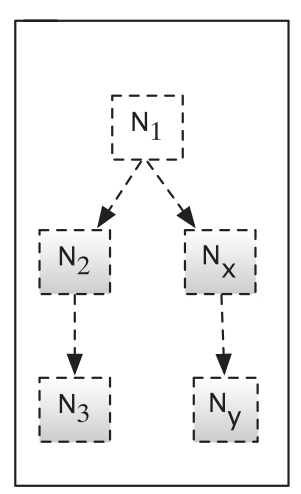

3

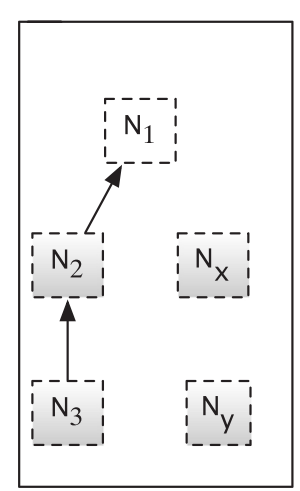

4

Fig. 5 Network topology of the application presented in Fig. 1. Each box represents the node in the network. The shaded nodes are new recipients. The dashed lines show possible reverse routes, while the solid lines show the discovered route. 1 Node N1 receives a low-priority binding request. $\mathbf{2} \mathrm{N} 1$ broadcasts a route discovery message, N2 and Nx receive it. $\mathbf{3} \mathrm{N} 2$ and Nx are not the intended destination. N2 and Nx make an entry in their node and forwards the request to their neighbours. 4 The intended destination N3 has been found. N3 sends out a route reply message along the reversed path of the request. This route reply message configures all the intermediate nodes and the source node 
The mobile robot application provides the greatest opportunities for data aggregation due to its high data rate and one-hop network structure, while the smart office application is more challenging. In reality, we expect the characteristics of most WSN applications to fall somewhere between those of these two applications. The smart office and the mobile robots applications are overlaid with a non-critical node health monitoring application, which uses low-priority Hitch Hiker bindings, described in Section 7.3.

\subsection{Smart office application}

The smart office application aims to ensure employee comfort, while reducing energy consumption by sensing environmental conditions and controlling relevant appliances. Sensor nodes $\left(N_{2}, N_{3}\right.$ and $\left.N_{4}\right)$ monitor: temperature, light and whether the window is open or closed every $30 \mathrm{~s}$. This sensor data is transmitted to a comfort level component running on the cluster-head node $\left(N_{1}\right)$ which aggregates the sensor information and forwards the aggregated data to a manager component running on a server $\left(N_{0}\right)$, once every $30 \mathrm{~s}$. Based upon the observed sensor data and configured comfort levels, a management component running on the server $\left(N_{0}\right)$ issues commands to a control component running on the cluster-heads $\left(N_{1}\right)$, which then activate or deactivate relay switches running on nodes $N_{2}$ to $N_{4}$ that control: lighting, ventilation and an audio alarm, which indicates that the window should be closed. The smart office application is realised using high-priority (i.e. standard) LooCI bindings. The payload size of all sensor data is $\mathbf{4}$ bytes, the payload size of aggregated data is 12 bytes and the payload size of relay control commands is 4 bytes.

Figure 6 shows the application composition and all relevant binding and properties information. In terms of network topology, the scenario is comprised of 25 offices.
Each office contains three sensor nodes, and a clusterhead node. Sensor nodes communicate with cluster heads, which in turn communicate with a single server for management of comfort level. This approximates a 101-node tree topology rooted at the server.

\subsection{Mobile robot application}

The mobile robot application coordinates a set of mobile robots to detect chemical spills. Each robot $\left(N_{1}\right.$ to $\left.N_{100}\right)$ runs a chemical sensor and a location sensor which sample every $10 \mathrm{~s}$ and transmit the data to a coordination component running on the server $\left(N_{0}\right)$. The coordination component then calculates a set of navigation instructions every $10 \mathrm{~s}$ and transmits these to the navigation component running on each mobile robot $\left(N_{1}\right.$ to $\left.N_{100}\right)$. The mobile robot application is realised using standard LooCI bindings. The payload size of location data is 6 bytes, the payload size of chemical sensor data is 4 bytes and the payload size of navigation commands is 5 bytes. Figure 6 shows the application composition and all relevant binding and properties data. In terms of network topology, the scenario contains 100 mobile robots, all of which communicate directly with the coordinating server. This approximates a 101-node star topology.

\subsection{Node health monitoring application}

The node health monitoring application [35] is a lowpriority application, inspired from real world deployments such as Great Duck Island [36]. We consider it low-priority because it adds value, but (i.) the data is not critical and (ii.) it should not reduce the lifetime of the base application. This component monitors battery level, memory use and the radio link quality. The application consists of a health monitor component that runs on all sensor nodes $\left(N_{1}\right.$ to $\left.N_{n}\right)$ and sends node health information to an alert component running on

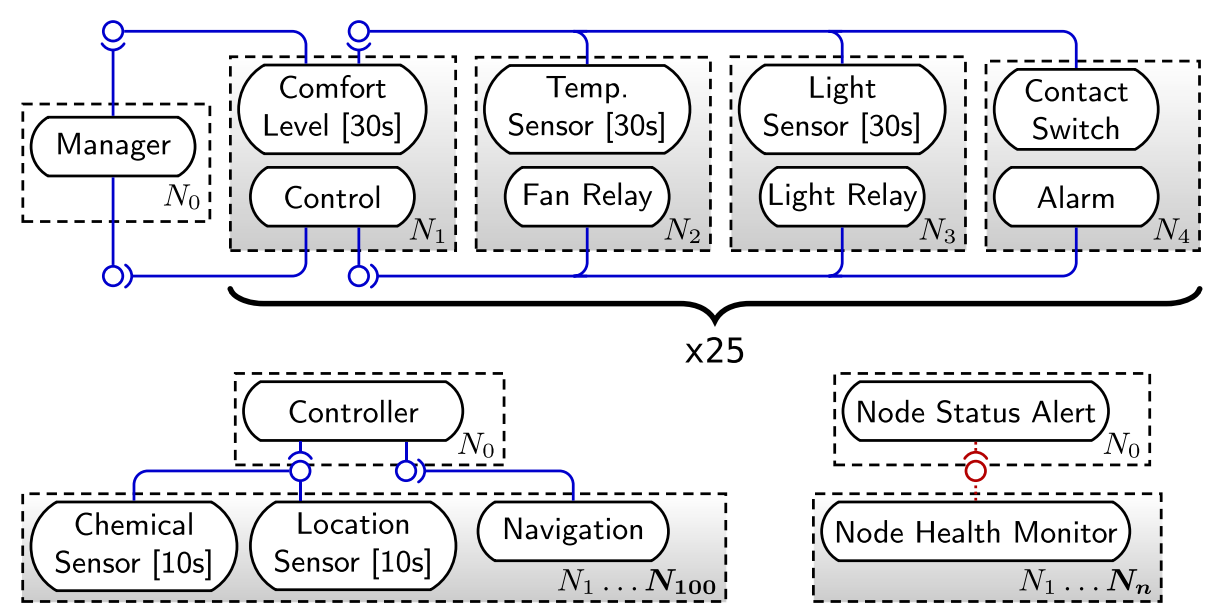

Fig. 6 Component bindings of the smart building (top), mobile robot (bottom-left) and monitoring (bottom-right) applications 
the server node $\left(N_{0}\right)$. Node health monitoring is overlaid on the smart building and mobile robot application using low-priority bindings. The payload size of node health monitor data is $18 \mathrm{~B}$. The composition is shown in Fig. 6.

\section{Implementation and evaluation}

We have developed prototypes of Hitch Hiker-2.0 for the $\mathrm{OMNeT}++$ simulator [17] and the Zigduino mote [37]. Simulation is used to study the performance of the three case-study applications described in Section 7. The Zigduino implementation validates node-local memory and energy characteristics for a concrete hardware/software stack.

OMNeT++ settings: The physical layer is a CC2420 IEEE 802.15.4 radio [38]. We use B-MAC [39] as a representative Low Power Listening (LPL) protocol. Simulation settings are based on prior experiments by Polastre et al. [39]. Table 2 shows the configuration settings of OMNeT++.

Zigduino configuration: Zigduino is an Arduinocompatible mote based on the ATmega128RFA1 [40], which offers a $16 \mathrm{MHz}$ MCU, $16 \mathrm{~KB}$ of RAM, $128 \mathrm{~KB}$ of Flash and an IEEE 802.15.4 radio. We use ContikiOS v2.6, Contiki X-MAC (CX-MAC) [41] and LooCI v2.0 [13] extended with Hitch Hiker 2.0. The parameterisation of CX-MAC uses the default Contiki values. In the case of the mobile robot case-study the Zigduino is extended with a ShieldBot mobile robot base [42]. Table 2 shows the configuration settings of Zigduino. We compare Hitch Hiker against (i.) transmission of standard messages (referred as Standard binding) and (ii.) an optimally configured one-hop data aggregation scheme using an optimal aggregation buffer size of 3 (referred as One-hop aggregation). Values reported below represent averages taken over one week.

All source code and simulation material are available at: http://goo.gl/7m2nwN.

\subsection{OMNET++ simulation results}

Latency Figure 7 shows the results of our latency simulation. The $x$-axis shows the sampling frequency of the node health monitoring app, which was set to a consistent fraction of the case study application frequency (from left to right from 10 to $50 \%$ of the base app frequency). The $y$ axis shows the latency of message transmission in seconds

Table 2 Configuration of the OMNeT++ simulation and the Zigduino implementation

\begin{tabular}{llllll}
\hline Radio & OMNet & Zigduino & MAC protocol & OMNet & Zigduino \\
\hline Transmit current & $17 \mathrm{~mA}$ & $18.6 \mathrm{~mA}$ & Check interval & $0.1 \mathrm{~s}$ & $0.125 \mathrm{~s}$ \\
Receive current & $16.2 \mathrm{~mA}$ & $16.6 \mathrm{~mA}$ & Slot duration & $1.0 \mathrm{~s}$ & $2.0 \mathrm{~s}$ \\
Sleep current & $0.02 \mathrm{~mA}$ & $4.1 \mathrm{~mA}$ & Queue length & 10 & 15 \\
\hline
\end{tabular}

for low-priority Hitch Hiker bindings, standard bindings and one-hop data aggregation.

As expected, the node health monitoring app exhibited a higher latency when using low-priority bindings than with standard bindings due to packets waiting for aggregation at each hop. However, the latency of low-priority bindings is lower than the one-hop aggregation scheme due to the exploitation of multi-hop routes. For the mobile robot app (right of Fig. 7) the latency of Hitch Hiker falls as high-priority traffic is transmitted at a higher rate and thus, there are increased opportunities for aggregation.

Energy Figure 8 shows the results of our energy simulation. As with our latency experiments, the sampling frequency of the node health monitoring app was set to 10 to $50 \%$ of the base application frequency. The $y$-axis shows the power consumption low-priority Hitch Hiker bindings, standard bindings and one-hop data aggregation.

The results shown in Fig. 8 confirm the expected savings when using Hitch Hiker to route low-priority traffic. Energy consumption is reduced by up to $15 \%$ in the smart building scenario and up to $32 \%$ in the mobile robot scenario compared to standard bindings. The energy consumption of Hitch Hiker is also lower than that of one-hop data aggregation. The greatest savings are achieved for the mobile robot application as more low-priority transmissions are aggregated.

\subsection{Zigduino/Contiki implementation results}

This section reports the performance timings of route configuration and message transmission as well as energy consumption and memory overhead for the Contiki/Zigduino implementation. Configuration timings are dependent upon the type of route being configured. We therefore report average timings based upon the smart building application, as this has the most complex routing structure.

Route Creation The Hitch Hiker 2.0 provides two approaches for route creation. Infrastructure Hitch Hiker requires approximately $86 \mathrm{~ms}$ to configure a single lowpriority binding. Each additional hop that must be configured adds $30 \mathrm{~ms}$ to the configuration overhead. Route configuration is thus lightweight; creating all of the Hitch Hiker bindings required for the smart building takes less than $3 \mathrm{~s}$. However, this generates three transmissions per Hitch Hiker binding, which costs $36.5 \mathrm{~mJ}$.

In contrast, Ad-hoc Hitch Hiker, takes more time to configure a route since it uses data aggregation overlay itself to flood route discovery messages. For the smart building application, in the worst case, Ad-hoc Hitch Hiker requires $65 \mathrm{~s}$ to configure a single low priority binding. Each additional hop that must be configured adds $30 \mathrm{~s}$ 

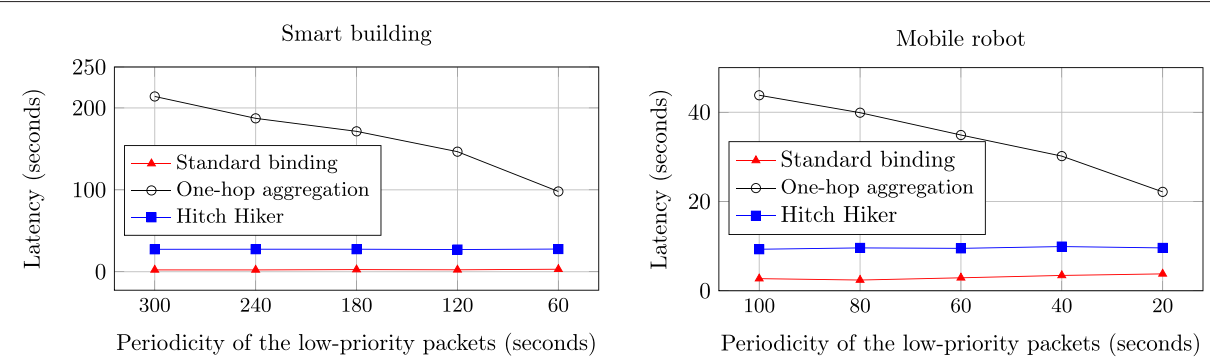

Fig. 7 Latency of the health monitoring app. overlaid on the smart building (left) and mobile robot (right), with Hitch Hiker-2.0, one hop aggregation and standard bindings

to the configuration time, and the complete smart building app takes less than an hour to configure. However, Ad-hoc Hitch Hiker generates only two transmissions per Hitch Hiker binding, which costs $23 \mathrm{~mJ}$ - significantly less than infrastructure mode.

Message Transmission Enqueueing, dequeueing and encapsulating a single Hitch Hiker packet within a host frame requires on average of $12.27 \mathrm{~mJ}$, while a standard frame transmission using CX-MAC requires $21.41 \mathrm{~mJ}$, a saving of $57.4 \%$ compared to standard transmission.

Memory Hitch Hiker introduces minimal memory overhead in comparison to the basic LooCI component model. As shown in Table 3, the implementation of Infrastructure Hitch Hiker adds $3 \%$ of ROM and $8 \%$ of RAM to the LooCI component model. This implementation consists of component model probe along with Hitch and Hiker protocols, as presented in Section 3.

For Ad-hoc Hitch Hiker, the ROM and RAM overhead is approximately 6 and $8 \%$ respectively. Ad-hoc Hitch Hiker consumes more memory than Infrastructure mode as each node must embed route discovery logic. Each routing table entry uses an additional $6 \mathrm{~B}$ of memory. We believe that this low overhead is reasonable in light of the energy savings reported in Section 8.1.

\section{Conclusions and future work}

This paper introduced Hitch Hiker 2.0, a novel remote binding model for IoT which supports prioritised bindings and multi-hop data aggregation. This prioritised binding model provides developers with a low-effort mechanism to manage data aggregation. Unlike prior work in the area of aggregation, Hitch Hiker uses component binding meta-data to construct a multi-hop overlay network for data aggregation. Hitch Hiker provides support for routing on multi-hop overlay network. To the best of our knowledge, Hitch Hiker is both the first generic and yet application aware data aggregation approach. Furthermore, Hitch Hiker is the first remote binding model to provide built-in support for data aggregation.

Hitch Hiker 2.0 extended our previous work [14] and provides a decentralised routing approach. Hitch Hiker 2.0 allows the user to choose between centralised and decentralised routing approach, making it a flexible binding model.

We have simulated the Hitch Hiker protocol in $\mathrm{OMNeT}++$ for two case-study application scenarios. Our results show that using Hitch Hiker to route low-priority traffic reduces energy consumption and, for applications with a high data rate, latency. We also implemented a prototype of Hitch Hiker for the LooCI component model running on the Contiki OS and the Zigduino mote. Our evaluation of the prototype implementation shows that Hitch Hiker consumes minimal memory, introduces limited overhead and that transmitting
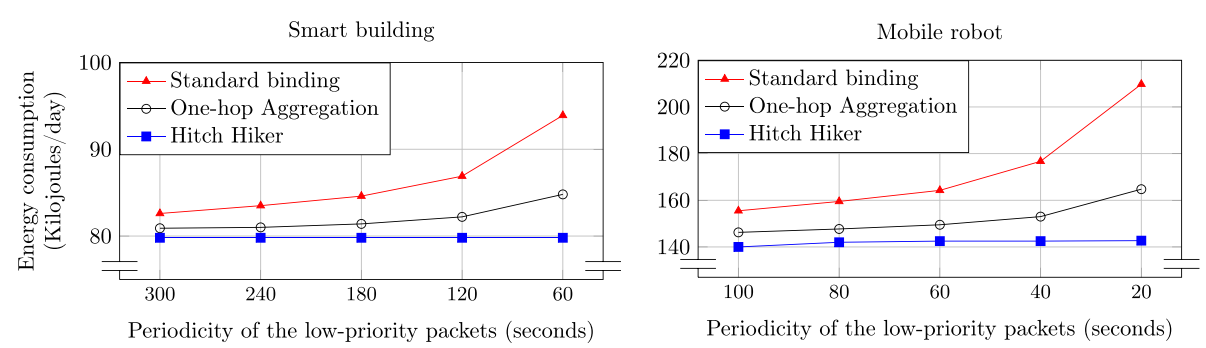

Fig. 8 Energy consumption of health monitoring overlaid on the smart building (left) and mobile robot (right), with Hitch Hiker-2.0, one-hop aggregation and standard bindings 
Table 3 Memory overhead of Hitch Hiker-2.0 (HH)

\begin{tabular}{llll}
\hline Memory & LooCl & Infrastructure HH & Ad-Hoc HH \\
\hline ROM & 56534 B & $1882 \mathrm{~B}(+3.3 \%)$ & $3244 \mathrm{~B}(+5.7 \%)$ \\
RAM & 8998 B & $722 \mathrm{~B}(+8.0 \%)$ & $756 \mathrm{~B}(+8.4 \%)$ \\
\hline
\end{tabular}

messages with Hitch Hiker consumes a small fraction of the energy that is required for a standard radio transmission.

Our future work will focus on four fronts: improving the performance of Hitch Hiker for non-periodic components, adding support for virtual circuits, extending Hitch Hiker to support variable component payloads and realising a RPC version of the Hitch Hiker binding model.

Non-periodic components: The current design of Hitch Hiker tends to avoid aggregation with non-periodic bindings, where the source component not specify the rate property, due to the unpredictability performance of those links. This is a potential source of inefficiency in cases where non-periodic components transmit frequently. We plan to address this inefficiency by extending the Component Model Probe with support for monitoring the transmission timings of non-periodic components and extracting timing data.

Virtual circuits: In the current model, it is possible for the Hitch Hiker overlay to become congested and for buffers to overflow. In our future work, we will explore how resource reservation can be used to create virtual circuits on top of the Hitch Hiker overlay with associated Quality of Service assurances. We envisage that this could be achieved by extending the role of the network manager to include remote configuration of Hitch buffer sizes and admission control on low-priority bindings.

Variable component payloads: The current design of Hitch Hiker supports only fixed sized data types. While we believe that this covers the vast majority of WSN traffic, it is interesting to explore how Hitch Hiker could be extended to support variable sized payloads such as compressed images or microphone captures. As with non-periodic components this would necessitate extension of the Component Model Probe to support the monitoring of previous transmissions and maintenance of historic payload size data.

Remote Procedure Call: As Hitch Hiker extends LooCI, it supports only unidirectional event-based bindings. It would be interesting to extend Hitch Hiker with support for Remote Procedure Call (RPC) bindings. As RPC method calls are inherently request-reply and therefore bidirectional, this would result in a much more densely connected data aggregation overlay and therefore improved performance for Hitch Hiker.

\section{Competing interests}

The authors declare that they have no competing interests.

\section{Authors' contributions}

GSR worked on the design, implementation and the evaluation of Hitch Hiker. JP worked on the design and modeling. WD helped with the implementation on Zigduino hardware platform. MP,DS,CH and WJ were involved in the discussions during the design phase. $\mathrm{DH}$ supervised and played a critical role in the entire work. In addition, all authors read and approved the work.

\section{Acknowledgements}

This research is partially supported by the Research Fund, KU Leuven and iMinds (a research institute founded by the Flemish government), and by the Portuguese FCT grant SFRH/BPD/91908/2012. The research is conducted in the context of FWO-RINAiSense project.

\section{Author details}

${ }^{1}$ iMinds-DistriNet, KU Leuven, 3001 Leuven, Belgium. ${ }^{2}$ iMinds-IBCN, Ghent University, 9000 Gent, Belgium. ${ }^{3}$ HASLab/INESC TEC, Universidade do Minho, Braga, Portugal.

Received: 2 September 2015 Accepted: 23 April 2016

Published online: 05 May 2016

\section{References}

1. Raghunathan V, Schurgers C, Park S, Srivastava M, Shaw B. Energy-aware wireless microsensor networks. In: IEEE Signal Processing Magazine. IEEE; 2002. p. 40-50.

2. Rajagopalan R, Varshney PK. Data-aggregation techniques in sensor networks: A survey. IEEE Commun Surv Tutor. 2006;8(4):48-63. doi:10.1109/COMST.2006.283821.

3. Tan HO, Körpeoğlu I. Power efficient data gathering and aggregation in wireless sensor networks. SIGMOD Rec. 2003;32(4):66-71. doi:10.1145/959060.959072.

4. Kalpakis K, Dasgupta K, Namjoshi P. Efficient algorithms for maximum lifetime data gathering and aggregation in wireless sensor networks. Comput Netw. 2003;42(6):697-716. doi:10.1016/S1389-1286(03)00212-3.

5. He T, Blum BM, Stankovic JA, Abdelzaher T. Aida: Adaptive applicationindependent data aggregation in wireless sensor networks. ACM Trans Embed Comput Syst. 2004426-457. doi:10.1145/993396.993406.

6. Intanagonwiwat C, Govindan R, Estfin D, Heidemann J, Silva F. Directed diffusion for wireless sensor networking. IEEE/ACM Trans Networking. 2003;11:2-16.

7. Madden S, Franklin MJ, Hellerstein JM, Hong W. Tag: A tiny aggregation service for ad-hoc sensor networks. SIGOPS Oper Syst Rev. 2002131-146. doi:10.1145/844128.844142.

8. Heinzelman WR, Chandrakasan A, Balakrishnan H. Energy-efficient communication protocol for wireless microsensor networks. In: 33rd Annual Hawaii Int. Conf. on System Sciences; 2000. p. 10. doi:10.1109/HICSS.2000.926982.

9. Aonishi T, Matsuda T, Mikami S, Kawaguchi H, Ohta C, Yoshimoto M. Impact of aggregation efficiency on git routing for wireless sensor networks. In: Int. Conf. on Parallel Processing Workshops; 2006. p. 8-158. doi:10.1109/ICPPW.2006.41.

10. Birrell AD, Nelson BJ. Implementing remote procedure calls. ACM Trans Comput Syst. 1984. 39-59. doi:10.1145/2080.357392.

11. May TD, Dunning SH, Dowding GA, Hallstrom JO. An RPC design for wireless sensor networks. Int J Pervasive Comput Commun. 2007;2(4): 384-397.

12. von Eicken T, Culler DE, Goldstein SC, Schauser KE. Active messages: A mechanism for integrated communication and computation. In: 19th Annual Int. Symposium on Computer Architecture; 1992. p. 256-266. doi:10.1145/139669.140382.

13. Hughes D, Thoelen K, Maerien J, Matthys N, Del Cid J, Horre W, Huygens C, Michiels S, Joosen W. Looci: The loosely-coupled component infrastructure. In: IEEE Symposium on Network Computing and Applications; 2012. p. 236-243. doi:10.1109/NCA.2012.30.

14. Ramachandran GS, Daniels W, Proença J, Michiels S, Joosen W, Hughes D, Porter B. Hitch hiker: A remote binding model with priority based data 
aggregation for wireless sensor networks. In: Proceedings of the 18th International ACM SIGSOFT Symposium on Component-Based Software Engineering. CBSE '15. New York: ACM; 2015. p. 43-48. doi:10.1145/2737166.2737179. http://doi.acm.org/10.1145/2737166. 2737179.

15. Perkins $C E$, Royer EM. Ad-hoc on-demand distance vector routing. In: Mobile Computing Systems and Applications; 1999. p. 90-100. doi:10.1109/MCSA.1999.749281.

16. Dunkels A, Gronvall B, Voigt T. Contiki - a lightweight and flexible operating system for tiny networked sensors. In: 29th Annual IEEE Int. Conf. on Local Computer Networks; 2004. p. 455-462. doi:10.1109/LCN.2004.38.

17. Chen K. Performance Evaluation by Simulation and Analysis with Applications to Computer Networks. NJ, USA: Wiley; 2015.

18. He T, Stankovic JA, Lu C, Abdelzaher T. Speed: a stateless protocol for real-time communication in sensor networks. In: Distributed Computing Systems, 2003. Proceedings. 23rd Int. Conf. On; 2003. p. 46-55. doi:10.1109/ICDCS.2003.1203451.

19. Heinzelman WR, Kulik J, Balakrishnan H. Adaptive protocols for information dissemination in wireless sensor networks. In: 5th Annual ACM/IEEE Int. Conf. on Mobile Computing and Networking. New York: ACM; 1999. p. 174-185. doi:10.1145/313451.313529.

20. Asemani $M$, Esnaashari M. Learning automata based energy efficient data aggregation in wireless sensor networks. Wirel Netw. 2015;21(6): 2035-2053. doi:10.1007/s11276-015-0894-3.

21. Kalpakis K, Dasgupta K, Namjoshi P. Efficient algorithms for maximum lifetime data gathering and aggregation in wireless sensor networks. Comput Netw. 2003;42(6):697-716. doi:10.1016/S1389-1286(03) 00212-3.

22. Xue $Y$, Cui $Y$, Nahrstedt K. Maximizing lifetime for data aggregation in wireless sensor networks. Mob Netw Appl. 2005;10(6):853-864. doi:10.1007/s11036-005-4443-7.

23. Voulkidis AC, Anastasopoulos MP, Cottis PG. Energy efficiency in wireless sensor networks: A game-theoretic approach based on coalition formation. ACM Trans Sen Netw. 2013;9(4):43-14327. doi:10.1145/2489253. 2489260.

24. Xiang L, Luo J, Rosenberg C. Compressed data aggregation: Energy-efficient and high-fidelity data collection. IEEE/ACM Trans Netw. 2013;21(6):1722-1735. doi:10.1109/TNET.2012.2229716.

25. Krishnamachari B, Estrin D, Wicker $S$. The impact of data aggregation in wireless sensor networks. In: 22nd Int. Conf. on Distributed Computing Systems Workshops; 2002. p. 575-578 doi:10.1109/ICDCSW.2002.1030829.

26. Villas LA, Boukerche A, Ramos HS, de Oliveira HABF, de Araujo RB, Loureiro AAF. Drina: A lightweight and reliable routing approach for in-network aggregation in wireless sensor networks. IEEE Trans Comput. 2013;62(4):676-689. doi:10.1109/TC.2012.31.

27. Jeong J, Kim J, Cha W, Kim H, Kim S, Mah P. A qos-aware data aggregation in wireless sensor networks. In: Advanced Communication Technology (ICACT), 2010 The 12th International Conference On. IEEE; 2010. p. 156-161.

28. Tavares ALC, Valente MT. A gentle introduction to OSGi. SIGSOFT Softw Eng Notes. 2008;33(5):8-185. doi:10.1145/1402521.1402526.

29. Costa P, Coulson G, Mascolo C, Picco GP, Zachariadis S. The runes middleware: a reconfigurable component-based approach to networked embedded systems. In: IEEE 16th Int. Symposium on Personal, Indoor and Mobile Radio Communications; 2005. p. 806-8102. doi:10.1109/PIMRC.2005. 1651554

30. Gay D, Levis P, von Behren R, Welsh M, Brewer E, Culler D. The nesc language: A holistic approach to networked embedded systems. In: ACM Conf. on Programming Language Design and Implementation; 2003. p. 1-11. doi:10.1145/781131.781133.

31. Simon $D$, Cifuentes $C$. The squawk virtual machine: Java ${ }^{\mathrm{TM}}$ on the bare metal. In: Companion to the 20th Annual ACM SIGPLAN Conference on Object-oriented Programming, Systems, Languages, and Applications. OOPSLA '05. New York: ACM; 2005. p. 150-151. doi:10.1145/1094855.1094908. http://doi.acm.org/10.1145/1094855. 1094908.

32. Thoelen K, Preuveneers D, Michiels S, Joosen W, Hughes D. Types in their prime: Sub-typing of data in resource constrained environments In: Stojmenovic I, Cheng Z, Guo S, editors. Mobile and Ubiquitous Systems: Computing, Networking, and Services. Lecture Notes of the Institute for
Computer Sciences, Social Informatics and Telecommunications Engineering. New York: Springer; 2014. p. 250-261.

33. Tanenbaum AS. Computer Networks (4. Ed.) NJ, USA, Prentice Hall; 2002. pp. -1891 .

34. Shelby Z, Bormann C. 6LoWPAN: The Wireless Embedded Internet. NJ, USA: Wiley Publishing; 2010.

35. Werner-Allen G, Lorincz K, Ruiz M, Marcillo O, Johnson J, Lees J, Welsh M. Deploying a wireless sensor network on an active volcano. IEEE Internet Comput. 2006;10(2):18-25. doi:10.1109/MIC.2006.26.

36. Szewczyk R, Mainwaring A, Polastre J, Anderson J, Culler D. An analysis of a large scale habitat monitoring application. In: 2 nd Int. Conf. on Embedded Networked Sensor Systems. SenSys '04; 2004. p. 214-226. doi:10.1145/1031495.1031521.

37. Logos Electromechanical. Zigduino Manual. 2014. Logos Electromechanical. Rev. 2. http://www.logoselectro.com/ documentation/.

38. Texas Instruments. CC2420 Datasheet. 2014. Texas Instruments. http:// www.ti.com/product/CC2420

39. Polastre J, Hill J, Culler D. Versatile low power media access for wireless sensor networks. In: 2nd Int. Conf. on Embedded Networked Sensor Systems. SenSys '04. New York: ACM; 2004. p. 95-107. doi:10.1145/1031495.1031508.

40. Atmel Corporation. ATmega128RFA1 Datasheet. 2012. Atmel Corporation. http://www.atmel.com/devices/atmega1284.aspx.

41. Buettner M, Yee GV, Anderson E, Han R. X-MAC: A short preamble MAC protocol for duty-cycled wireless sensor networks. In: 4th Int. Conf. on Embedded Networked Sensor Systems; 2006. p. 307-320. doi:10.1145/1182807.1182838.

42. Seeedstudio. Shield Bot. 2014. Seeedstudio. http://www.seeedstudio com/wiki/Shield_Bot.

\section{Submit your manuscript to a SpringerOpen ${ }^{\circ}$ journal and benefit from:}

- Convenient online submission

- Rigorous peer review

- Immediate publication on acceptance

- Open access: articles freely available online

- High visibility within the field

- Retaining the copyright to your article

Submit your next manuscript at springeropen.com 